\title{
Analysis of Density Wave Oscillations in Helically Coiled Tube Once-Through Steam Generator
}

\author{
Junwei Hao, ${ }^{1}$ Yaoli Zhang, ${ }^{1}$ Jianxiang Zheng, ${ }^{1}$ Zhiwei Zhou, ${ }^{2}$ Xuanyu Sheng, ${ }^{2}$ Gang Hong, \\ Kai Ye, ${ }^{1}$ and $\mathrm{Ning} \mathrm{Li}^{1}$ \\ ${ }^{1}$ College of Energy, Xiamen University, Xiamen, China \\ ${ }^{2}$ Institute of Nuclear and New Energy Technology, Tsinghua University, Beijing, China
}

Correspondence should be addressed to Yaoli Zhang; zhangyl@xmu.edu.cn

Received 3 September 2016; Revised 1 November 2016; Accepted 6 November 2016

Academic Editor: Alejandro Clausse

Copyright (C) 2016 Junwei Hao et al. This is an open access article distributed under the Creative Commons Attribution License, which permits unrestricted use, distribution, and reproduction in any medium, provided the original work is properly cited.

Helically coiled tube Once-Through Steam Generator (H-OTSG) is one of the key equipment types for small modular reactors. The flow instability of the secondary side of the H-OTSG is particularly serious, because the working condition is in the range of low and medium pressure. This paper presents research on density wave oscillations (DWO) in a typical countercurrent H-OTSG. Based on the steady-state calculation, the mathematical model of single-channel system was established, and the transfer function was derived. Using Nyquist stability criterion of the single variable, the stability cases were studied with an in-house computer program. According to the analyses, the impact law of the geometrical parameters to the system stability was obtained. RELAP5/MOD3.2 code was also used to simulate DWO in H-OTSG. The theoretical analyses of the in-house program were compared to the simulation results of RELAP5. A correction factor was introduced to reduce the error of RELAP5 when modeling helical geometry. The comparison results agreed well which showed that the correction is effective.

\section{Introduction}

A steam generator is a type of heat exchanger specifically designed to transfer heat from a coolant into water, producing steam that can be utilized for power generation. Helically coiled tube steam generators are widely used in nuclear industry for their compactness, easy manufacture, and enhanced heat transfer efficiency [1]. They are widely adopted in different plants, such as HTGR, SMR, and IRIS. The most prominent characteristic of flow in helically coiled tubes is the secondary flow induced by centrifugal force due to the curvature of the pipe, thus resulting in significantly larger heat transfer and friction factors with respect to straight pipes.

In steam generators, extensive attention is attached to flow instabilities, as they can lead to mechanical vibrations, thermal fatigue, problems of system control, and heat transfer surface burnout issues [2]. Hence, it is imperative to avoid flow instabilities and determine the safe operating regions of steam generators through rational design and accurate definition of the threshold values of system parameters. DWO is the most representative instability encountered in boiling systems. It is well known that DWO is induced in boiling system by the interaction between the single-phase flow and two-phase flow pressure drops, the inlet mass flow rate, and the void fraction distribution [3].

Two-phase flow instabilities including DWO have been studied since the 1960s; many researchers contributed to understand the instability mechanisms, namely, Yadigaroglu and Bergles [4], Ishii [5], and Fukuda and Kobori [6]. Numerous theoretical and experimental works are collected in several excellent literature reviews $[7,8]$. Previous and current researches on flow instabilities mainly focus on straight tubes, whereas the research on helically coiled tubes has received much less attention. Most helically coiled tube correlations are formed based on straight pipe correlations by introducing a correction factor to adapt to the new geometry. Zhou et al. [9] and Ju et al. [10] carried out experiments to study DWO in helically coiled tube steam generators. More recently, experimental and theoretical studies were performed by Papini et al. [3] to investigate DWO in single and 
two parallel helically coiled tubes. Frequency domain method was employed to evaluate DWO in HTR-10 in the study of Niu et al. [11]. Most studies with respect to flow instabilities have been limited to constant wall temperature or constant wall heat flux conditions, using electrically heated tubes to assure uniform power distribution. The simplification provides a more convenient way to study the influence of each parameter on flow instability phenomena. However, the power distribution in a practical steam generator is not uniform; the electrically heated conditions will bring in some differences.

The present work deals with a fluid to fluid helically coiled steam generator, where neither constant wall temperature nor constant wall heat flux conditions can be assumed. Therefore, the results are more applicable to engineering practice. The methods to model two-phase flow instabilities fall into two categories: time domain method and frequency domain method. The frequency domain method is inexpensive with respect to computer time and it is less susceptible to numerical stability problems, so it is chosen in this work to evaluate the stability of helically coiled tubes.

Small modular reactors are becoming the research focus of nuclear industry in China currently. H-OTSG is adopted in many small modular reactors, such as HTR-10 (THU), ACP100S (CNNC), and ACPR50S (CGN). The flow instability of the secondary side in H-OTSG is a common problem to be solved in all these reactors. This work deals with a typical H-OTSG, focusing on the investigation of the influence of geometrical parameters of helically tubes on instability occurrence comprehensively with an in-house code, which can offer effective guidance to the design of H-OTSG. The H-OTSG model was also built in RELAP5/MOD3.2 as a comparison to authenticate each other.

\section{Models and Correlations}

2.1. Description of the Code. A FORTRAN code, STFQX (STEAMFREQ-X), was developed by Chan [12] to predict DWO in liquid-sodium steam generators, using linear frequency domain methods. The stability predictions were in satisfactory agreement with the experimental data. The simulations in this work were performed on the basis of STFQX. In STFQX, sodium flows outside the pipe while water flows inside, representing the steam generator in countercurrent fashion. The secondary side is divided into five sections:

(1) subcooled unheated region, which includes piping, pump, and channel inlet resistance,

(2) heated subcooled region,

(3) boiling region,

(4) superheated region,

(5) outlet resistance and adiabatic riser.

The analysis is versatile and allows any of the above parts that are absent in the system to be eliminated.

The STFQX code is applicable for three geometries: straight tubes, helical tubes, and serpentine tubes; however, correlations in the code are only valid for straight tubes. Helical tubes are represented by inclined straight tubes of the same length and inclination. The differences between helical tubes and straight tubes are ignored in this approximation, and it will bring in some deviations when modeling helically coiled configurations. In order to analyze DWO in helical tubes more precisely, heat transfer and pressure drop correlations in STFQX were modified. The modified STFQX code is named STFQH. New correlations dedicated to helical tubes are employed in STFQH, taking the effects of the centrifugal forces into account. The key geometrical parameters of helical tubes are the tube length, the tube inner diameter, the helix angle, the helix diameter, and wall thickness. Besides, the fluid properties in single-phase region are assumed to be constant in the original code, neglecting their variations with temperature. In STFQH, the fluid properties in subcooled region and superheated region, including density, heat capacity, viscosity, and thermal conductivity, are modified to be the function of temperature in the steady-state solution.

Assumptions are used as follows in the development of the model:

(1) The homogenous flow model is assumed in boiling region.

(2) One-dimensional model along the axis of tube is adopted.

(3) The heat conduction in the axial direction is neglected.

(4) The tube wall physical properties are assumed to be constant.

(5) Fluid properties do not vary with pressure and enthalpy perturbations.

(6) The flow and heat transfer parameter of every control unit are represented by the mean values.

(7) Subcooled boiling is neglected.

\subsection{Correlations for H-OTSG}

2.2.1. Heat Transfer Correlations. Heat transfer correlations for H-OTSG are listed in Table 1, which are cited from Cao's thesis [13]. The heat transfer coefficients in helically coiled pipes are modified on the basis of straight pipe correlations with correction factors. The heat transfer coefficients in pipes are closely related to the flow patterns. Laminar and turbulent flows are the two major forms of flow in straight tubes. In helical tubes, the flow conditions are affected by the centrifugal forces which separate the liquid and gas phases due to the density difference. De is the dimensionless Dean number, which accounts for the effects of secondary flow induced by centrifugal forces in helical tubes. Transition from laminar flow to turbulent flow is governed by the critical Reynolds number $\mathrm{Re}_{\mathrm{cr}}$ suggested by Cioncolini and Santini [14].

The flow patterns in helical tubes are divided into three types considering the effects of Reynolds number and Dean number:

(1) When $\mathrm{De}<11.6, \mathrm{Re}<\mathrm{Re}_{\mathrm{cr}}$, it is laminar flow.

(2) When $\mathrm{De}>11.6, \mathrm{Re}<\mathrm{Re}_{\mathrm{cr}}$, it is laminar flow with big vortex.

(3) When $\operatorname{Re}>\operatorname{Re}_{c r}$, it is turbulent flow. 
TABLE 1: Heat transfer correlations in the tube side and shell side of H-OTSG.

Correlations

Experimental parameters

Tube side [13]

Single-phase region:

$\mathrm{Nu}= \begin{cases}0.15 \operatorname{Re}^{0.33} \operatorname{Pr}^{0.43}\left(\frac{\operatorname{Pr}}{\operatorname{Pr}_{w}}\right)^{0.25} & \mathrm{Re} \leq \mathrm{Re}_{\mathrm{cr}}, \quad \mathrm{De} \leq 11.6 \\ 0.06 \mathrm{De}^{0.37} \operatorname{Re}^{0.33} \operatorname{Pr}^{0.43}\left(\frac{\operatorname{Pr}}{\operatorname{Pr}_{w}}\right)^{0.25} & \mathrm{Re} \leq \mathrm{Re}_{\mathrm{cr}}, \quad \mathrm{De}>11.6 \\ 0.023 \operatorname{Re}^{0.8} \operatorname{Pr}^{0.4}\left[1+6.3\left(1-\frac{d}{D}\right)\left(\frac{d}{D}\right)^{1.15}\right] & \mathrm{Re}>\mathrm{Re}_{\mathrm{cr}}\end{cases}$

$0.1<P<20 \mathrm{MPa}$

$80<G<3000 \mathrm{~kg} /\left(\mathrm{m}^{2} \cdot \mathrm{s}\right)$

Critical Reynolds number [14]:

$\operatorname{Re}_{\mathrm{cr}}= \begin{cases}2300\left[1+210\left(\frac{D}{d}\right)^{-1.12}\right] & \frac{D}{d} \geq 150 \\ 12500\left(\frac{D}{d}\right)^{-0.31} & 30 \leq \frac{D}{d} \leq 110 \\ 30000\left(\frac{D}{d}\right)^{-0.47} & \frac{D}{d} \leq 24\end{cases}$

$6.9<\frac{D}{d}<369$

Nucleate boiling region:

$h=h_{M} \sqrt{1+7 \times 10^{-9}\left(\frac{0.7 h_{0}}{h_{M}}\right)^{2}\left(\frac{u_{m} \rho_{f} h_{f g}}{q}\right)^{1.5}}$

$h_{M}=\sqrt{h_{f}^{2}+\left(0.7 h_{0}\right)^{2}}$,

$h_{f}=0.021 \frac{\lambda_{f}}{d} \operatorname{Re}_{f}{ }^{0.85} \operatorname{Pr}^{0.4}\left(\frac{d}{D}\right)^{0.1}$,

$h_{0}=3.13 q^{0.7}\left(0.12 P^{0.14}+1.9 \times 10^{-14} P^{2}\right)$

Postdryout region:

$\mathrm{Nu}= \begin{cases}0.017 \theta \operatorname{Re}_{\mathrm{cm}}{ }^{0.8} \operatorname{Pr}^{0.8} & \frac{D}{d}<66.7 \\ 0.023 \operatorname{Re}_{\mathrm{cm}}{ }^{0.8} \operatorname{Pr}^{0.8}\left[1-0.1\left(\frac{\rho_{f}}{\rho_{g}}-1\right)^{0.4}(1-x)^{0.4}\right] & \frac{D}{d} \geq 66.7\end{cases}$

$\theta=1+Y(1-x)$

$Y= \begin{cases}0.5\left(\frac{\rho_{f}}{\rho_{g}}-1\right) & \frac{\rho_{f}}{\rho_{g}} \leq 480 \\ 70 & \frac{\rho_{f}}{\rho_{g}}>480,\end{cases}$

$0.1<P<21.5 \mathrm{MPa}$

$100<G<2000 \mathrm{~kg} /\left(\mathrm{m}^{2} \cdot \mathrm{s}\right)$

$0<x<1$

$\operatorname{Re}_{\mathrm{cm}}=\frac{G d}{\mu_{g}}\left[x+\frac{\rho_{g}}{\rho_{f}}(1-x)\right]$

Shell side [13]:

$\mathrm{Nu}= \begin{cases}0.56 \operatorname{Re}^{0.5} \operatorname{Pr}^{0.33}\left(\frac{\operatorname{Pr}}{\operatorname{Pr}_{w}}\right)^{0.25} & 100<\operatorname{Re}<1000 \\ 0.2 \operatorname{Re}^{0.65} \operatorname{Pr}^{0.33}\left(\frac{\operatorname{Pr}}{\operatorname{Pr}_{w}}\right)^{0.25} & 1000<\operatorname{Re}<2 \times 10^{5}, \sigma>2 \\ \frac{0.2 \operatorname{Re}^{0.65}}{\left[1+3(1-\sigma / 2)^{3}\right]^{2}} \operatorname{Pr}^{0.33}\left(\frac{\operatorname{Pr}}{\operatorname{Pr}_{w}}\right)^{0.25} & 1000<\operatorname{Re}<2 \times 10^{5}, \sigma \leq 2 \\ 0.02 \operatorname{Re}^{0.84} \operatorname{Pr}^{0.33}\left(\frac{\operatorname{Pr}}{\operatorname{Pr}_{w}}\right)^{0.25} & \operatorname{Re}>2 \times 10^{5}\end{cases}$

$0.1<P<20 \mathrm{MPa}$

$80<G<3000 \mathrm{~kg} /\left(\mathrm{m}^{2} \cdot \mathrm{s}\right)$

$60<q<800 \mathrm{~kW} / \mathrm{m}^{2}$

$6.9<\frac{D}{d}<50$

$0.1<P<20 \mathrm{MPa}$

2.2.2. Pressure Drop Correlations. The pressure drop in the pipe consists of three parts: the frictional pressure drop, the gravitational pressure drop, and the acceleration pressure drop. The difference between pressure drop in helical pipe and straight pipe lies in the frictional pressure drop. Ju et al. correlations [10] are recommended in calculating frictional pressure drop in small bending radius helical pipe, as listed in Table 2.

\section{Theories of Stability Analysis}

3.1. Physical Process. DWO is reported to be the most commonly observed flow instability phenomenon. Mechanisms of DWO have been investigated in numerous literatures. Inlet flow perturbations in a heated channel result in delayed mixture density changes throughout the channel. These disturbances in the mixture density affect the local mixture 
TABLE 2: Frictional pressure drop correlations in H-OTSG.

Correlations

Experimental parameters

Single-phase region [10]:

When

$\mathrm{De}<11.6, \mathrm{Re}<\mathrm{Re}_{\mathrm{cr}}$,

$f_{s}=\frac{64}{\operatorname{Re}}$,

$\frac{f_{c}}{f_{s}}=1$

When

De $>11.6, \operatorname{Re}<\operatorname{Re}_{c r}$,

$f_{s}=\frac{64}{\operatorname{Re}}$

$\frac{f_{c}}{f_{s}}=1+0.015 \operatorname{Re}^{0.75}\left(\frac{d}{D}\right)^{0.4}$

$2.5<P<4.5 \mathrm{MPa}$

$200<G<1500 \mathrm{~kg} /\left(\mathrm{m}^{2} \cdot \mathrm{s}\right)$

When

$8.0<\frac{D}{d}<9.3$

$\operatorname{Re}>\operatorname{Re}_{\mathrm{cr}}$,

$f_{s}= \begin{cases}\frac{0.316}{\operatorname{Re}^{0.25}} & \text { smooth pipe } \\ 0.1\left(1.46 \frac{\Delta}{d}+\frac{100}{\operatorname{Re}}\right)^{0.25} & \text { rough pipe, }\end{cases}$

$\frac{f_{c}}{f_{s}}=1+0.11 \operatorname{Re}^{0.23}\left(\frac{d}{D}\right)^{0.14}$

Two-phase region [10]:

$\Delta P_{f}=f \frac{L}{d} \frac{\rho_{f} u_{0}^{2}}{2}\left[1+x\left(\frac{\rho_{f}}{\rho_{g}-1}\right)\right] \psi$,

$\psi=\left(1.29+A_{n} x^{n}\right)\left\{1+x\left[\left(\frac{\mu_{g}}{\mu_{f}}\right)^{0.25}-1\right]\right\}$,

$2.5<P<4.5 \mathrm{MPa}$

$200<G<1500 \mathrm{~kg} /\left(\mathrm{m}^{2} \cdot \mathrm{s}\right)$

$8.0<\frac{D}{d}<9.3$

where $A_{1}=2.19, A_{2}=-3.61, A_{3}=7.35, A_{4}=-5.93$

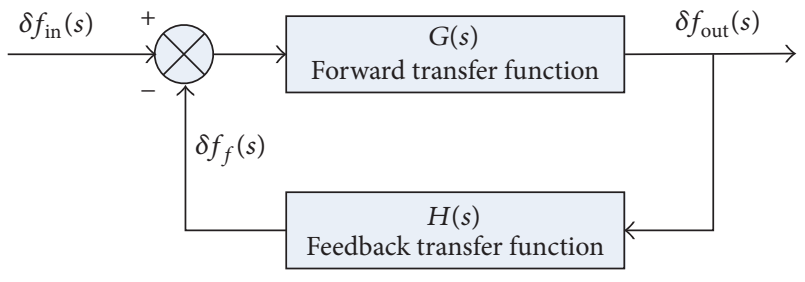

FIgURE 1: Schematic of a feedback control system.

velocity and the total pressure drop in a channel. Under certain conditions, the inlet flow perturbations and the internal pressure drop perturbations satisfy a self-exciting relation such that sustained oscillations with considerable amplitudes appear in the system [5]. This flow instability due to density wave propagations is so-called DWO. The selfexciting characters of DWO make a feedback system, which is shown in terms of the block diagram given in Figure 1. If a forcing function, $\delta f_{\text {in }}$, creates a feedback perturbation, the system is capable of self-exciting oscillations when $\delta f_{\text {in }}+$ $\delta f_{f}=0$, that is, $\delta f_{\text {in }} \cdot[1+G(s) \cdot H(s)]=0$, where $\delta f_{f}$ is the feedback perturbation. System stability is therefore independent of the forcing function.
3.2. Control Theory. According to the linear system control theory, linear system instability occurs when the system becomes self-exciting. The stability of a linear system can be determined in the frequency domain by utilizing Nyquist stability criterion. The Nyquist criterion is widely used in electronics and control system engineering, as well as other fields, for designing and analyzing systems with feedback. Nyquist is a graphical technique; it provides intuition for whether a system is stable or unstable and how to modify an unstable system to be stable. The stability of a closedloop system is determined from its open-loop frequency response and open-loop poles. The closed-loop system is unstable for any clockwise encirclement of the point $(-1,0)$ in the complex plane. Provided that certain conditions are met, stability of the system is assured if the Nyquist plot of the variable $\delta f_{f} / \delta f_{\text {in }}$ does not encircle the $(-1,0)$ point in the complex plane.

\section{Results and Discussion}

4.1. System Parameters of H-OTSG. This work deals with a typical countercurrent helical pipe steam generator, and water serves as coolant in both primary and secondary side, as shown in Figure 2. Geometrical and operating parameters 


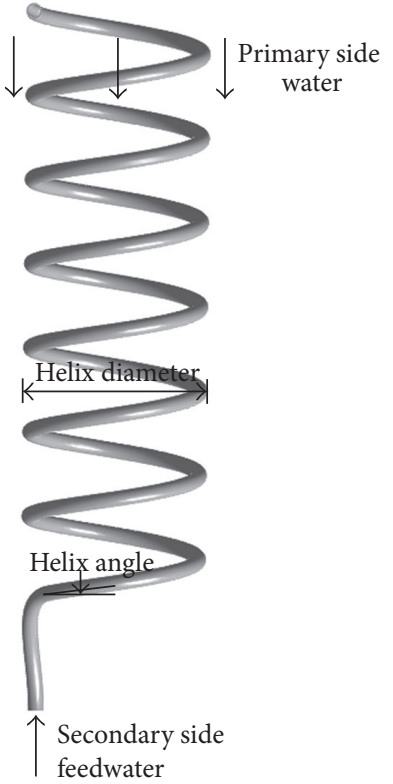

FIgURE 2: Structure and flow of H-OTSG.

TABle 3: Parameters for H-OTSG simulation in a single pipe.

\begin{tabular}{lc}
\hline Parameters & Values \\
\hline Inner diameter $(\mathrm{mm})$ & 14 \\
Wall thickness $(\mathrm{mm})$ & 2 \\
Helix diameter $(\mathrm{mm})$ & 112 \\
Helix angle $\left({ }^{\circ}\right)$ & 8 \\
Heated tube length $(\mathrm{m})$ & 27 \\
Primary side inlet pressure $(\mathrm{MPa})$ & 15.5 \\
Primary side inlet temperature $\left({ }^{\circ} \mathrm{C}\right)$ & 320.8 \\
Primary side flow rate $(\mathrm{kg} / \mathrm{s})$ & 2.0 \\
Feedwater pressure $(\mathrm{MPa})$ & 4 \\
Feedwater temperature $\left({ }^{\circ} \mathrm{C}\right)$ & 150.36 \\
Feedwater flow rate $(\mathrm{kg} / \mathrm{s})$ & 0.15 \\
\hline
\end{tabular}

are configured with reference to the existing reactors with helically coiled tube steam generators as a base case [15]. The main geometrical data and operating parameters are listed in Table 3.

4.2. Heat Flux Distribution of the Countercurrent H-OTSG. In the countercurrent steam generator, the heat flux changes along the tube, while the heat flux of uniform heating tube remains unchanged. The nonuniform heat transfer condition is a unique characteristic of this work, which is more applicable to engineering practice. The difference of heat flux distribution results in different flow conditions.

The heat flux along the tube in the typical countercurrent H-OTSG at full power condition is shown in Figure 3, compared with the uniform heating situation. Heat transfer quantities are the same in these two situations. In Figure 3, the secondary side feedwater flows from the inlet $($ location $=0)$ to the outlet (location $=27$ ) while the primary side water flows in the opposite direction. Regions 1, 2, 3, and 4 represent the

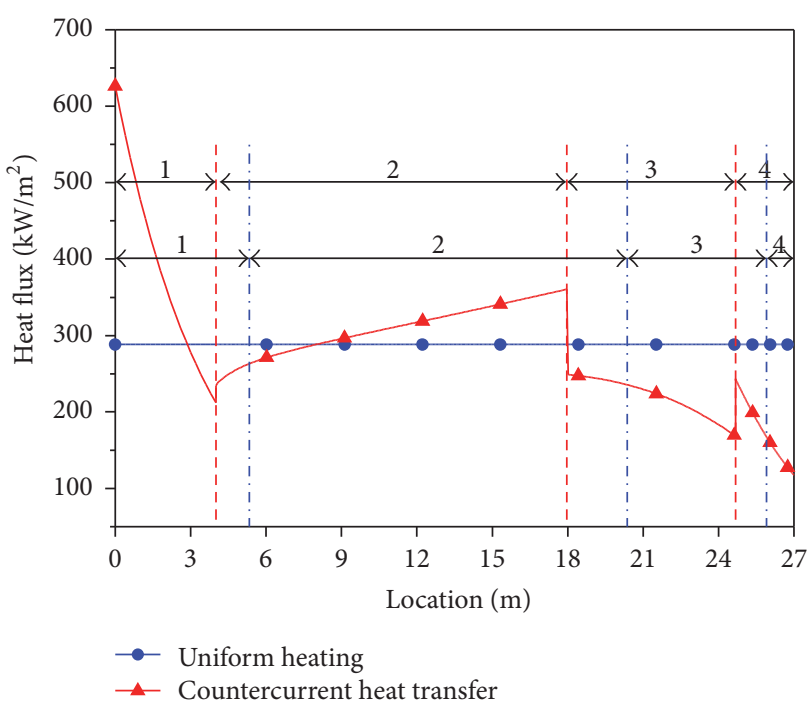

FIgURE 3: Heat flux along the helical tube in the countercurrent steam generator and uniform heating condition.

subcooled region, the nucleate boiling region, the postdryout region, and the superheated region, respectively, in these two situations. The heat flux changes dramatically in these four regions in the countercurrent situation. In the subcooled region, the heat flux is highest at the inlet and decreases gradually because of the temperature difference between the two sides. In the nucleate boiling region, the heat flux goes up with the increase of steam quality. The heat flux drops suddenly at the dryout point and goes down in the postdryout region. After a sudden increase at the boundary of postdryout region, the heat flux decreases continuously in the superheated region. The boundaries and lengths of all these four regions differ in the two situations, which reveal different flow and heat transfer conditions.

4.3. Parametric Effects on Flow Instability. The influence of main geometrical parameters of the countercurrent steam generator on system stability was investigated in the analysis with the STFQH code. The variation of any one of the parameters was performed in each case to understand how it affects the system stability.

4.3.1. Variation of Inlet Throttling. It is well known that inlet throttling, which offers a concentrated pressure drop located at the channel inlet, is an effective way of stabilizing. The Nyquist curves of different inlet throttling coefficients are shown in Figure 4(a); the inlet throttling pressure drops increase in proportion with throttling coefficients. The system becomes more stable when the Nyquist curve gets inside. When the curve has no clockwise encirclement of the point $(-1,0)$, DWO will not happen. Figure 4(a) confirms the effect of inlet throttling; DWO appears unless the inlet throttling coefficient becomes larger than 160. However, exaggerated inlet throttling will introduce a heavy burden to the pump load, which will reduce the efficiency of the power plant. 


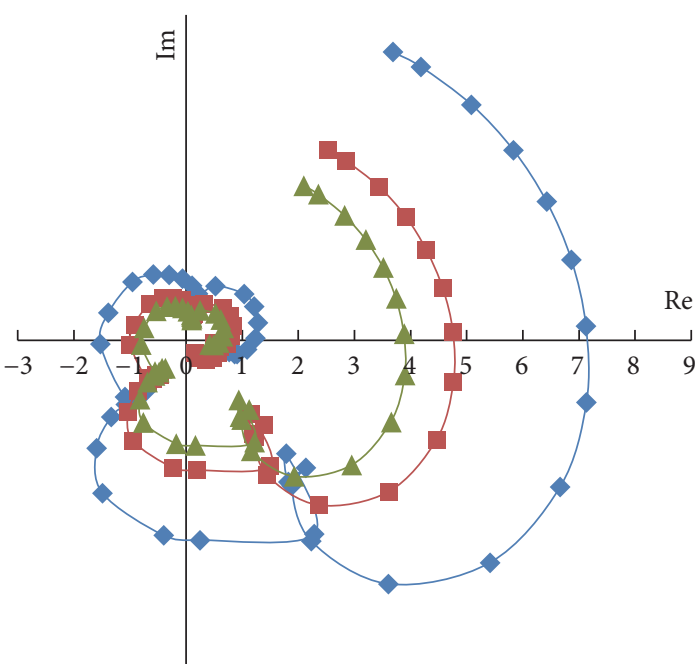

$\smile f=100$
$-f=160$
$-f=200$

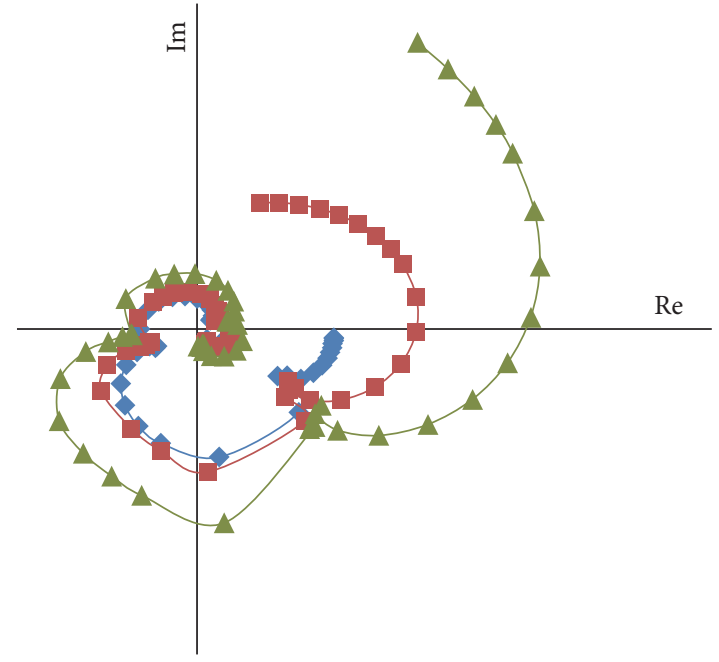

$\begin{aligned} \multimap L & =24 \mathrm{~m} \\ -L & =27 \mathrm{~m} \\ \multimap L & =30 \mathrm{~m}\end{aligned}$

(a)

(b)

Figure 4: Nyquist curves of the system. (a) Different inlet throttling coefficient. (b) Different heated length.

4.3.2. Variation of Heated Length. The system exhibits a marked stabilization when decreasing the length of channel. In Figure 4(b), three different tube lengths are presented: $24 \mathrm{~m}, 27 \mathrm{~m}$, and $30 \mathrm{~m}$. Obviously, the stabilization is smaller passing from $24 \mathrm{~m}$ to $30 \mathrm{~m}$. The inlet flow parameters are unique; a longer tube brings in larger heat transfer area, which will increase the heat transfer quantity and increase the exit steam quality eventually. Therefore, the longer pipe is expected to increase system propensity to flow instability.

Since the main goal of SG is to transfer a certain quantity of heat from the primary side to the secondary side, the influence of geometrical parameters with a certain heat transfer quantity was analyzed in the following cases. The heat transfer quantity was kept unchanged by adjusting the heated length. The same exit steam quality can be obtained due to the constant heat transfer quantity.

4.3.3. Variation of Helix Angle. In Figure 5(a), the Nyquist curves were obtained with varying helix angles while maintaining fixed heat transfer quantity. The system is prone to be stable when the helix angle increases. An increase in helix angle turns out a higher gravitational pressure drop term, concentrated in the subcooled region, where the fluid density is higher. The increase of single-phase region pressure drop, close to the channel inlet section, reinforces system stability.

4.3.4. Variation of Helix Diameter. Figure 5(b) reports how the helix diameter of heated channel affects DWO with a constant heat transfer quantity. The stronger secondary flow is caused by the greater centrifugal force when the helix diameter decreases, but it has only a limited impact on DWO as is shown in Figure 5(b). The variation of helix diameter is limited to the scope of the formula in this work; perhaps it will show a more significant effect when the helix diameter changes in a greater range.

4.3.5. Variation of Tube Inner Diameter. Three different Nyquist curves are depicted in Figure 5(c), corresponding to helically coiled tube inner diameter of $12 \mathrm{~mm}, 13 \mathrm{~mm}$, and $14 \mathrm{~mm}$, respectively. The heat transferred is set to a constant in the case. The mass velocity becomes larger and the heat transfer area becomes smaller with the decrease of the tube diameter. The larger mass velocity enhances the heat transfer and pressure drop while the smaller heat transfer area reduces it. The two opposite effects on heat transfer and pressure drop lead to a nonmonotonic transition of system stability as shown in Figure 5(c). Therefore, influence of tube diameter on DWO should be analyzed according to the specific configuration.

4.3.6. Variation of Wall Thickness. The present work models a fluid to fluid countercurrent steam generator; the decrease of wall thickness enhances the heat transfer between two sides and affects the heat transfer distribution along the tube. Figure 5(d) reports the influence of the variation of the wall thickness on DWO; the system becomes more stable as the wall thickness decreases. As the total heat transfer quantity is kept as a constant, the decrease of wall thickness results in a uniform decrease of thermal resistance along the tube. For the thermal resistance of two-phase region is smaller than singlephase region, the uniform decrease of thermal resistance that will reduce the heated length affects more two-phase region than single-phase region. Therefore, the proportion of twophase region decreases with the wall thickness. It appears 


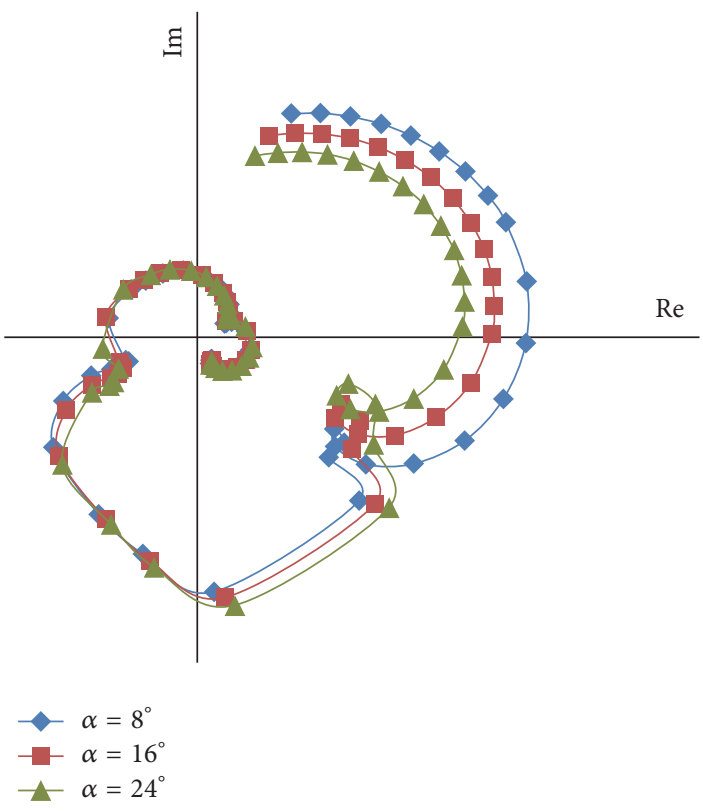

(a)

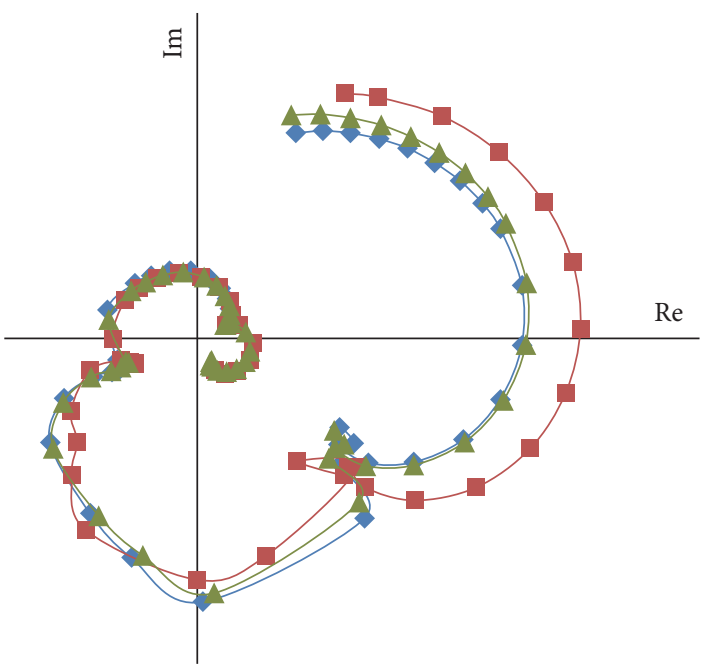

$\begin{aligned}-d & =12 \mathrm{~mm} \\ \rightarrow-d & =13 \mathrm{~mm} \\ -d & =14 \mathrm{~mm}\end{aligned}$

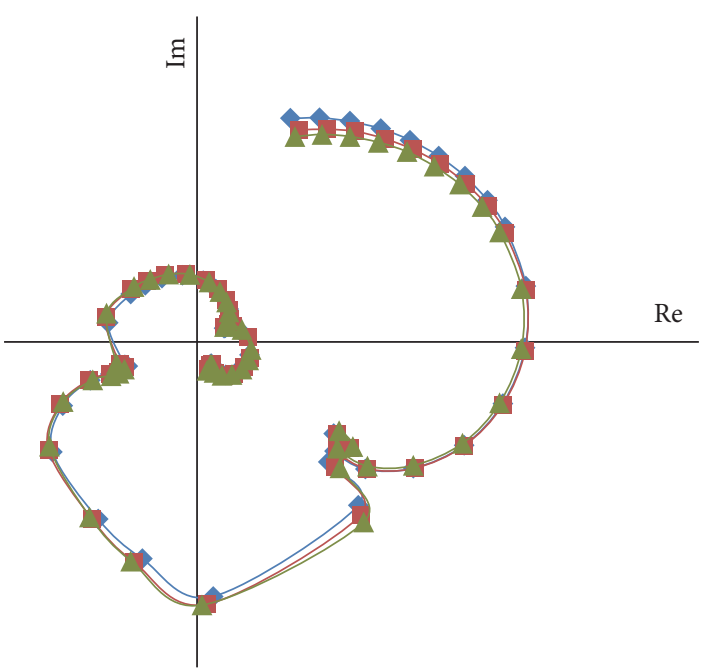

$\begin{aligned}-D & =112 \mathrm{~mm} \\ -D & =122 \mathrm{~mm} \\ -D & =132 \mathrm{~mm}\end{aligned}$

(b)

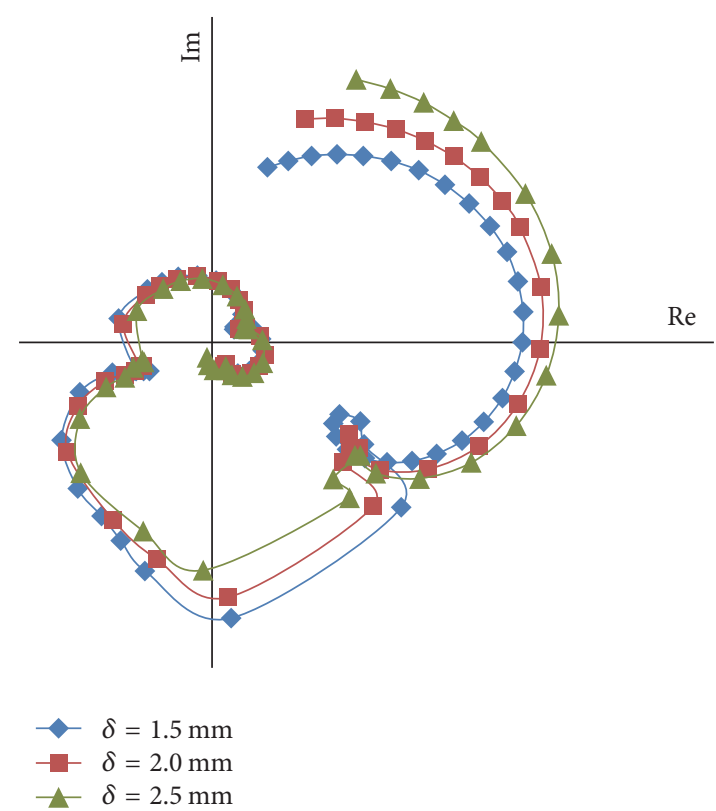

(d)

Figure 5: Nyquist curves of the system with a constant heat transfer quantity. (a) Different helix angle. (b) Different helix diameter. (c) Different tube inner diameter. (d) Different wall thickness.

that the decrease of proportion of two-phase region helps to stabilize the system. In addition to the effects on heat transfer area and flow instability, mechanical strength should be taken into consideration when choosing the tube wall thickness in the design of steam generator.

4.4. Comparisons between STFQH and RELAP5. It is well known that the RELAP5 code is usually considered for bestestimate transient simulation of thermal hydraulic phenomena relevant for light water reactor applications. The ability of
RELAP5 to analyze DWO has been confirmed in many works of literature $[3,16,17]$. RELAP5 and STFQH have respective advantages to analyze DWO in H-OTSG. STFQH has a higher computation efficiency than RELAP5, and the models of STFQH have been improved for helical pipes while all models in RELAP5 are for straight pipes. In addition, the flow instability of the system can be observed precisely by STFQH, whereas in RELAP5 simulation it may cause confusion with numerical instability. Despite the merits of STFQH, RELAP5 has its unique advantage. In RELAP5, DWO can be analyzed 


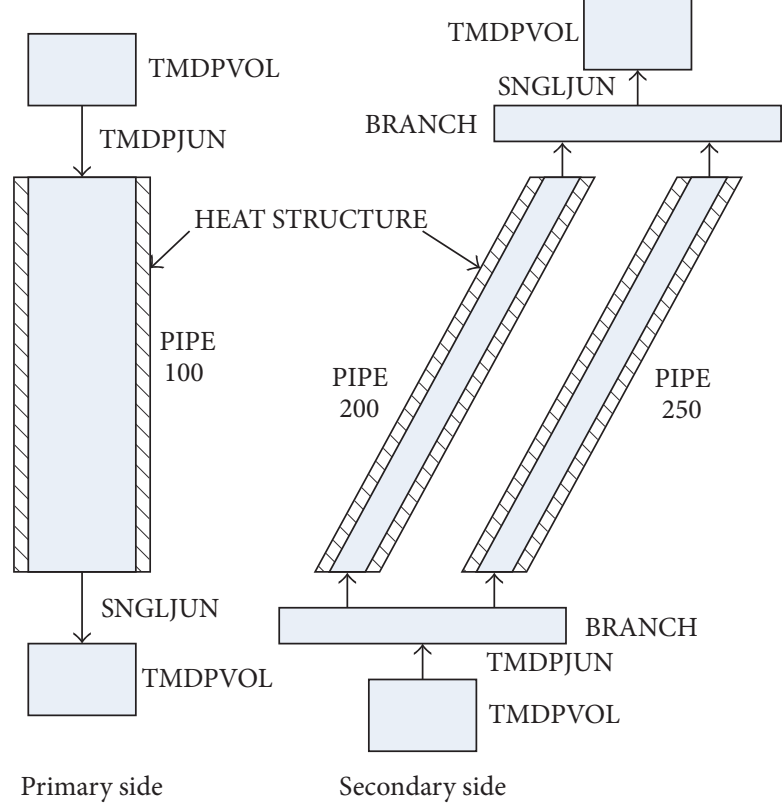

FIGURE 6: RELAP5 nodalization of a countercurrent H-OTSG with two parallel heated pipes.

integrally by connecting the H-OTSG to other facilities in RELAP5, and therefore it can be used for transient simulation under changed conditions, while STFQH can only be used in analysis of individual H-OTSG in steady state.

In this section, comparisons were made between STFQH and RELAP5 to verify and evaluate the ability of these two codes to predict DWO in H-OTSG. As is shown in Figure 6, two parallel pipes (PIPE 200 and PIPE 250) are connected to the same inlet and outlet plenum in the RELAP5 model, which represent the helical pipes. PIPE 100 represents the primary system of H-OTSG that transfers heat to PIPE 200 and PIPE 250 by the heat structure. The parameters of RELAP5 models are configured with reference to the base case described before. The general approximations, using an inclined pipe with the same length and inclination to represent the helical pipe, were adopted in the RELAP5 simulation. Same heat transfer areas, same gravitational pressure drops, and similar frictional pressure drops can be achieved in this hypothesis. The UVUT (Unequal Velocity Unequal Temperature) was chosen in this RELAP5 model, because it is more reliable compared with the EVET (Equal Velocity Equal Temperature) model. The semi-implicit numerical scheme was selected, as it is much less diffusive than the nearly implicit one [17]. A sensitivity study on the number of nodes used for the heated channel was conducted, with the number of nodes varying from 40 to 60 and 80.80 nodes were selected for higher accuracy in calculations.

As aforementioned, inlet throttling is effective in system stabilization. The threshold inlet throttling pressure drop, which exactly stabilizes the system, can be considered as a measurement criterion of the system instability. The threshold inlet throttling pressure drops to stabilize the system, as well as total pressure drops of heated region, were calculated

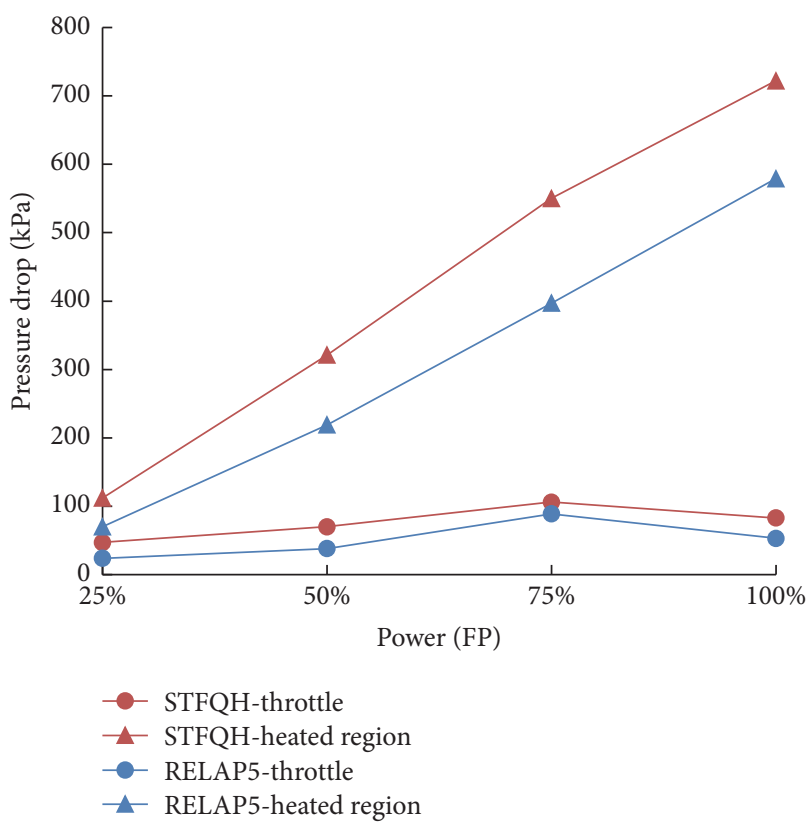

FIGURE 7: Comparisons between STFQH and RELAP5 regarding heated region pressure drops and threshold inlet throttling to stabilize the system.

by the two codes. Results at different power conditions from $25 \%$ FP to $100 \%$ FP are shown in Figure 7 . The heated region pressure drop of STFQH is $25 \%-60 \%$ larger than results from RELAP5 at different power conditions, because of the larger pressure drops in helical tubes than in straight tubes. The threshold inlet throttling pressure drop to stabilize the system is related to the total pressure drop in the heated region. Accordingly, the threshold inlet throttling pressure drops obtained from STFQH are larger than that from RELAP5.

To reduce the error brought by the helical pipe approximation of RELAP5, the threshold inlet throttling pressure drop obtained by RELAP5 is suggested to be multiplied by a correction coefficient. The correction coefficient is the ratio between the heated region pressure drops obtained by the two codes, which reveals the deviation between helical pipes and straight pipes. As is shown in Table 4, the error of threshold inlet throttling pressure drop between the corrected inclined pipe results from RELAP5 and the helical pipe results from $\mathrm{STFQH}$ is $-20 \%-17 \%$. The error is permissible, which verifies the abilities of STFQH and RELAP5 to predict DWO in HOTSG and proves the correction is reasonable.

\section{Conclusions}

The DWO phenomena of H-OTSG were analytically studied in this paper with the STFQH code, which utilizes single variable frequency domain method and has been suitably improved for helical geometry. Heat flux distribution of helical pipe in the fluid to fluid countercurrent H-OTSG was given, and it differs from that of the uniform heating pipe. Parametric effects of inlet throttling, heated length, helix angle, helix diameter, tube diameter, and wall thickness on 
TABLE 4: Comparisons between STFQH and corrected RELAP5 results concerning threshold inlet throttling pressure drops.

\begin{tabular}{lcccc}
\hline Power (FP) & Correction coefficient & $\begin{array}{c}\text { RELAP5 original pressure } \\
\text { drop (kPa) }\end{array}$ & $\begin{array}{c}\text { RELAP5 corrected pressure } \\
\text { drop (kPa) }\end{array}$ & \begin{tabular}{c} 
STFQH pressure drop (kPa) \\
\hline $25 \%$
\end{tabular} \\
$50 \%$ & 1.60 & 24 & 38.4 & 47 \\
$75 \%$ & 1.47 & 38 & 55.9 & 70 \\
$100 \%$ & 1.39 & 89 & 123.7 & 106 \\
\end{tabular}

DWO were analyzed based on different Nyquist curves. The situation of a constant heat transfer quantity was assumed in the analysis. These results, which enhance our knowledge of how various geometrical parameters affect the occurrence of DWO, could prove useful in guiding the design of H-OTSG. The simulation results of the fluid to fluid countercurrent $\mathrm{H}$ OTSG are more applicable to engineering practice compared with the studies which adopt uniform heating tubes.

The threshold inlet throttling pressure drops to stabilize the system and heat region pressure drops were compared between STFQH and RELAP5/MOD3.2. The pressure drops in helical pipes are larger than that in straight pipes under different power conditions. A correction coefficient was introduced in RELAP5 analysis to reduce the errors of stability threshold inlet throttling pressure drops brought by the approximation of helical pipes. The abilities of STFQH and RELAP5 to analyze DWO in H-OTSG were verified and the correction coefficients are proved to be reasonable.

\section{Nomenclature}

$d: \quad$ Inner tube diameter $(\mathrm{m})$

$d_{0}$ : Outer tube diameter $(\mathrm{m})$

D: Helix diameter $(\mathrm{m})$

De: Dean number, $\operatorname{De}=\operatorname{Re} \sqrt{d / D}(-)$

$f$ : Friction coefficient $(-)$

$f_{c}$ : Friction coefficient for helical pipe (-)

$f_{s}$ : Friction coefficient for straight pipe $(-)$

$G$ : Mass velocity $\left(\mathrm{kg} /\left(\mathrm{m}^{2} \cdot \mathrm{s}\right)\right)$

$h$ : Heat transfer coefficient $\left(\mathrm{W} / \mathrm{m}^{2} \cdot \mathrm{K}\right)$

L: $\quad$ Heated length $(\mathrm{m})$

$\mathrm{Nu}$ : Nusselt number $(-)$

p: Coil pitch $(\mathrm{m})$

$P$ : $\quad$ System pressure $(\mathrm{Pa})$

Pr: Prandtl number $(-)$

q: Heat flux $\left(\mathrm{kW} / \mathrm{m}^{2}\right)$

Re: Reynolds number (-)

$u_{0}$ : Flow velocity, supposing the pipe is full of liquid $(\mathrm{m} / \mathrm{s})$

$u_{m}$ : Mean velocity of water $(\mathrm{m} / \mathrm{s})$

$x$ : Steam quality $(-)$

\section{Greek Symbols}

$\alpha$ : Helix angle $\left({ }^{\circ}\right)$

$\rho$ : Density $\left(\mathrm{kg} / \mathrm{m}^{3}\right)$

$\lambda$ : Thermal conductivity $(\mathrm{W} / \mathrm{m} \cdot \mathrm{K})$

$\sigma$ : Relative distance, $\sigma=p / d_{0},(-)$

$\mu$ : Dynamic viscosity $(\mathrm{Pa} \cdot \mathrm{s})$

$\delta$ : Tube thickness $(\mathrm{m})$
$\Delta: \quad$ Roughness $(-)$

$\Delta P_{f}$ : Frictional pressure drop $(\mathrm{Pa})$

Subscripts

cr: Critical

$f$ : Saturated liquid

$g$ : Saturated vapour

$w$ : At wall temperature.

\section{Competing Interests}

The authors declare that there is no conflict of interests regarding the publication of this paper.

\section{Acknowledgments}

This work was supported by the Fundamental Research Funds for Xiamen University (20720150095) and the Foundation of Key Laboratory, Ministry of Education, China (ARES201402).

\section{References}

[1] A. M. Fsadni and J. P. M. Whitty, "A review on the twophase heat transfer characteristics in helically coiled tube heat exchangers," International Journal of Heat and Mass Transfer, vol. 95, pp. 551-565, 2016.

[2] M. Colombo, D. Papini, A. Cammi, and M. E. Ricotti, "Experimental investigation of thermal hydraulic instabilities in steam generator helical coil tubes," ENEA. Report, 2011.

[3] D. Papini, M. Colombo, A. Cammi, and M. E. Ricotti, "Experimental and theoretical studies on density wave instabilities in helically coiled tubes," International Journal of Heat and Mass Transfer, vol. 68, pp. 343-356, 2014.

[4] G. Yadigaroglu and A. Bergles, "An experimental and theoretical study of density-wave phenomena oscillations in twophase flow," Tech. Rep., Department of Mechanical Engineering Massachusetts Institute of Technology Cambridge, Cambridge, Mass, USA, 1969.

[5] M. Ishii, "Study on flow instabilities in two-phase mixtures," Argonne National Laboratory Report ANL-76-23, 1976.

[6] K. Fukuda and T. Kobori, "Classification of two-phase flow instability by density wave oscillation model," Journal of Nuclear Science and Technology, vol. 16, no. 2, pp. 95-108, 1979.

[7] S. Kakac and B. Bon, "A review of two-phase flow dynamic instabilities in tube boiling systems," International Journal of Heat and Mass Transfer, vol. 51, no. 3-4, pp. 399-433, 2008. 
[8] L. C. Ruspini, C. P. Marcel, and A. Clausse, "Two-phase flow instabilities: a review," International Journal of Heat and Mass Transfer, vol. 71, pp. 521-548, 2014.

[9] Y. L. Zhou, T. K. Chen, and X. J. Chen, “The prediction of twophase flow instability of stream generator in high-temperature gas-cooled reactor," Chinese Journal of Nuclear Science and Engineering, vol. 14, no. 2, pp. 97-103, 1994.

[10] H. Ju, Z. Huang, Y. Xu, B. Duan, and Y. Yu, "Hydraulic performance of small bending radius helical coil-pipe," Journal of Nuclear Science and Technology, vol. 38, no. 10, pp. 826-831, 2001.

[11] F. Niu, L. Tian, Y. Yu, R. Li, and T. L. Norman, "Studies on flow instability of helical tube steam generator with Nyquist criterion," Nuclear Engineering and Design, vol. 266, pp. 63-69, 2014.

[12] K. C. Chan, Thermal-hydraulic stability analysis of steam generators [Ph.D. thesis], University of California, Berkeley, Calif, USA, 1979.

[13] D. Cao, Thermal-technical analysis of once-through big helical tube steam generator [M.S. thesis], Tsinghua University, 2005.

[14] A. Cioncolini and L. Santini, "An experimental investigation regarding the laminar to turbulent flow transition in helically coiled pipes," Experimental Thermal and Fluid Science, vol. 30, no. 4, pp. 367-380, 2006.

[15] International Atomic Energy Agency, Status of Small and Medium Reactor Designs, IAEA, Vienna, Austria, 2012.

[16] M. Colombo, A. Cammi, D. Papini, and M. E. Ricotti, "RELAP5/MOD3.3 study on density wave instabilities in single channel and two parallel channels," Progress in Nuclear Energy, vol. 56, pp. 15-23, 2012.

[17] W. Ambrosini and J. C. Ferreri, "Analysis of basic phenomena in boiling channel instabilities with different flow models and numerical schemes," in Proceedings of the 14th International Conference on Nuclear Engineering (ICONE '14), Miami, Fla, USA, July 2006. 

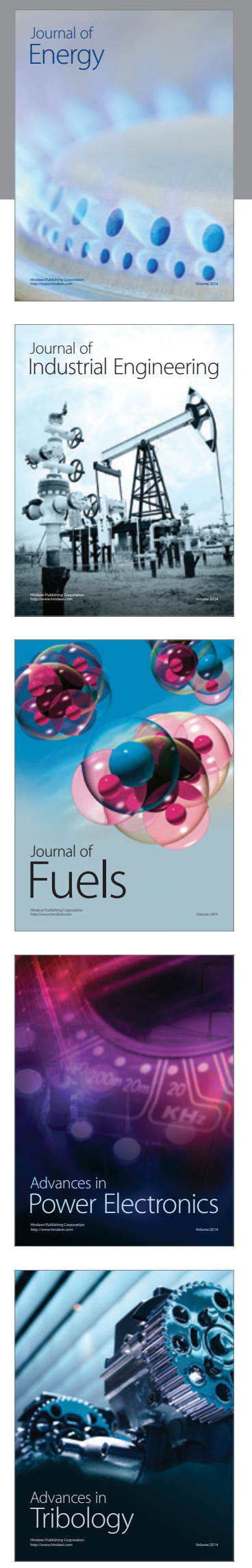
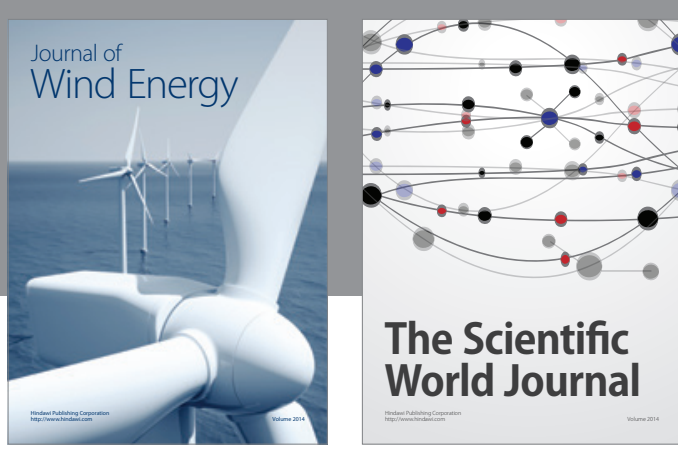

The Scientific World Journal
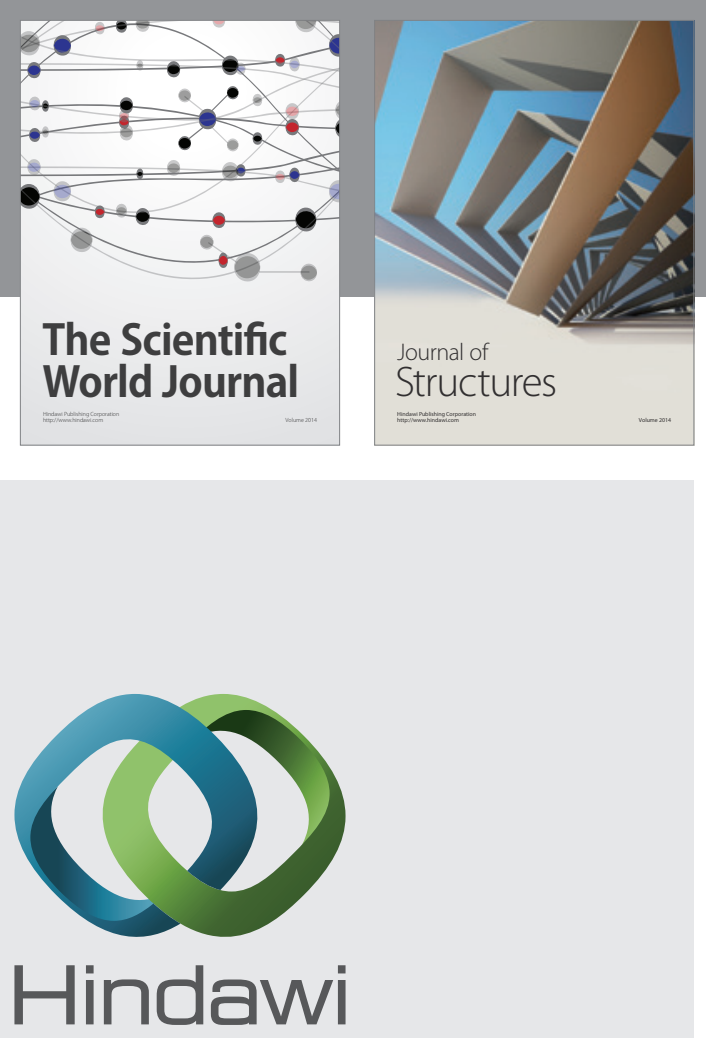

Submit your manuscripts at

http://www.hindawi.com
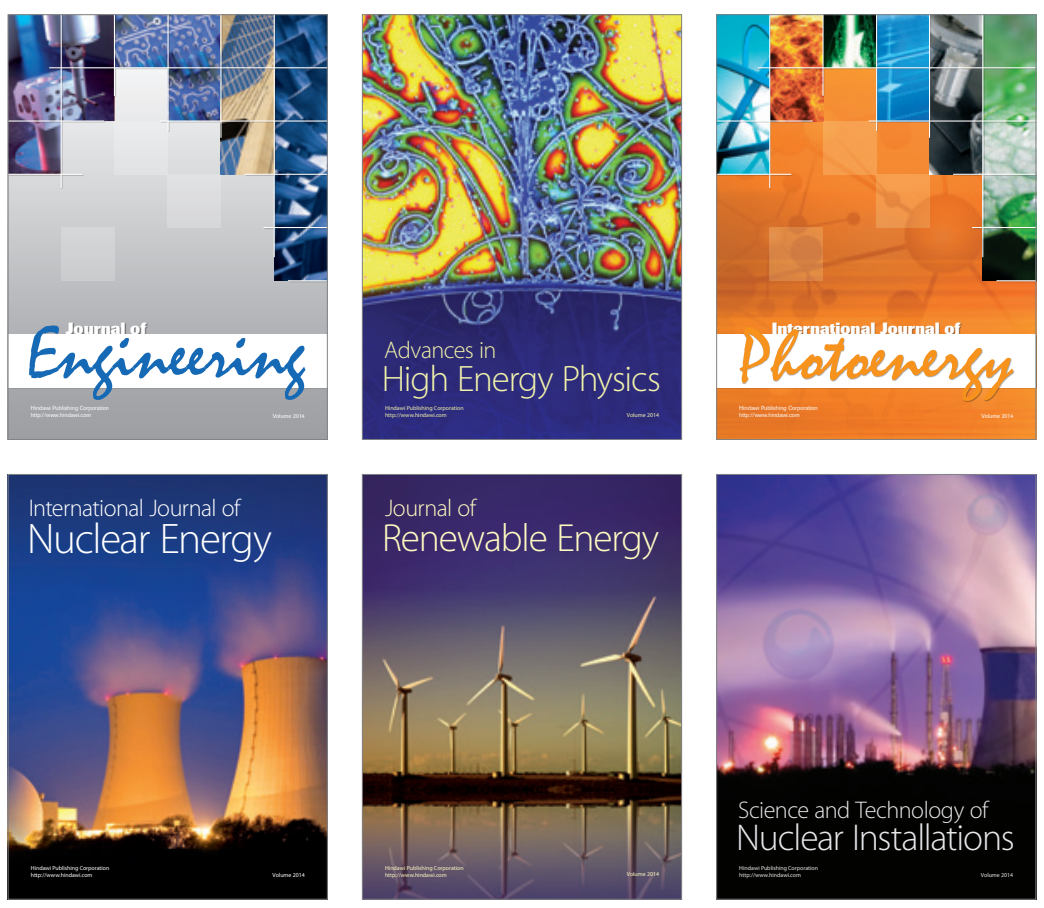
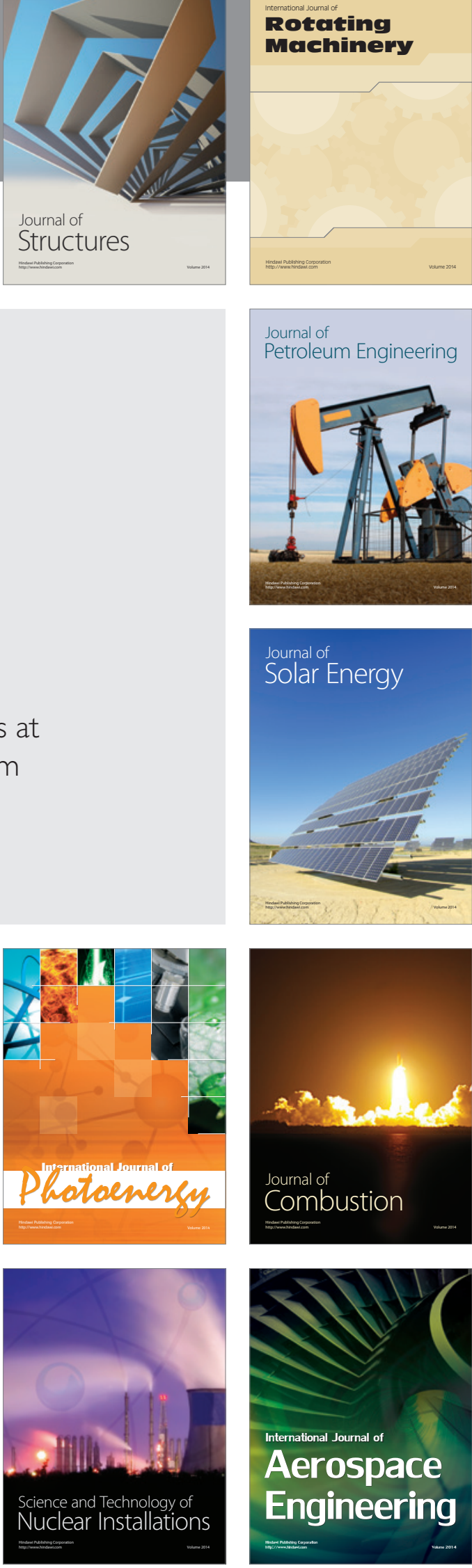